The ASTROPhySICAL Journal, 248:L13-L16, 1981 August 15

( 1 1981. The American Astronomical Society. All rights reserved. Printed in U.S.A.

\title{
VARIABLE POSITRON ANNIHILATION RADIATION FROM THE GALACTIC CENTER REGION
}

\author{
Guenter R. Riegler, James C. Ling, William A. Mahoney, William A. Wheaton, \\ JAMES B. WILLETT, AND ALLAN S. JACOBSON \\ Jet Propulsion Laboratory, California Institute of Technology \\ AND \\ Thomas A. Prince \\ California Institute of Technology \\ Received 1981 March 23; accepted 1981 May 6
}

\begin{abstract}
The HEAO 3 Cosmic Gamma-Ray Spectrometer performed the first high spectral resolution survey of the entire sky at gamma-ray energies from $50 \mathrm{keV}$ to $10 \mathrm{MeV}$. Studies of $511 \mathrm{keV}$ positron annihilation radiation from the vicinity of the galactic center are reported here, based on data which were recorded during 1979 September/October and 1980 March/April. The 1979 fall data show unshifted, narrow $511 \mathrm{keV}$ line emission of intensity $(1.85 \pm 0.21) \times 10^{-3}$ photons $\mathrm{cm}^{-2} \mathrm{~s}^{-1}$, consistent with earlier measurements. The 1980 spring measurement showed a statistically significant reduction in $511 \mathrm{keV}$ emission from this region, thus requiring that a significant fraction of the flux originate in one or more compact sources of size $\lesssim 10^{18} \mathrm{~cm}$. While distribution of sources within $\sim 22^{\circ}$ (at 90\% confidence level) of the direction of the galactic center are allowed by the observations, the data rule out most extended models for positron production, such as by cosmic ray interaction in the interstellar medium or by distributions of many supernovae, novae, or pulsars. The data are well satisfied by assuming that the emission originates in a single compact source at the galactic center.
\end{abstract}

Subject headings: galaxies: Milky Way — gamma rays: general

\section{INTRODUCTION}

Line emission in the gamma-ray energy range from 0.1 to $10 \mathrm{MeV}$ is produced in astrophysical sources by nuclear processes. This is in contrast to astronomical $\mathrm{X}$-ray emission where lines are generated by atomic transitions. The most prominent cosmic gamma-ray emission lines are expected to include the positronelectron annihiliation line at $511.003 \mathrm{keV}$, the deuterium line at $2.223 \mathrm{MeV}$, and prompt lines from proton bombardment of ${ }^{12} \mathrm{C}(4.438 \mathrm{MeV})$ and ${ }^{16} \mathrm{O}(6.129 \mathrm{MeV})$. These lines have been observed or predicted for the Earth's atmosphere, the Sun, interstellar grains, the interstellar gas, supernova remnants, novae, pulsars, black holes, and galaxy cores.

Positrons are expected to be produced via pair production and the decay of radioactive nuclei. Significant electron-positron pair production is expected to occur in the very strong magnetic fields $\left(\sim 10^{12}\right.$ gauss) that may exist around young neutron stars (Sturrock 1971). Annihilation near the neutron star surface could lead to gravitationally redshifted $511 \mathrm{keV}$ line emission; this radiation may have been detected already (Leventhal, MacCallum, and Watts 1977; Jacobson et al. 1978; Lingenfelter, Higdon, and Ramaty 1978; Teegarden and Cline 1980; Mazets et al. 1981).
Positron annihilation (Leventhal 1973; Bussard, Ramaty, and Drachman 1979) proceeds either directly or via the formation of positronium. The positron annihilation rate and the shape of the resulting photon emission spectrum near $511 \mathrm{keV}$ depend on the density, temperature, and degree of ionization in the annihilation region.

Reported observations of galactic $511 \mathrm{keV}$ line emission are summarized in Table 1. Scintillation detector observations, by Johnson and Haymes (1973) and Haymes et al. (1975), and germanium crystal measurements, by Leventhal, MacCallum, and Stang (1978), Leventhal et al. (1980), and Albernhe et al. (1981), resulted in line flux levels of $(0.8-4) \times 10^{-3}$ photons $\mathrm{cm}^{-2} \mathrm{~s}^{-1}$. None of these measurements could determine the spatial extent of the observed radiation and were therefore consistent with a variety of theoretical models.

\section{INSTRUMENTATION}

The HEAO 3 Gamma-Ray Spectrometer consisted of a cluster of four cooled germanium crystals which was surrounded by a CsI anticoincidence shield. The effective area of the four crystals at $511 \mathrm{keV}$ was $26.4 \mathrm{~cm}^{2}$. The average instrumental resolution at $511 \mathrm{keV}$ was 2.72 $\mathrm{keV}$ full width at half-maximum (FWHM) for the 1979 
TABLE 1

Positron ANNihilation Line Emission ObSERvations

\begin{tabular}{|c|c|c|c|}
\hline $\begin{array}{l}\text { Line Center } \\
\text { Energy (keV) }\end{array}$ & $\begin{array}{l}\text { Line Flux } \\
\left(10^{-3} \text { photons } \mathrm{cm}^{-2} \mathrm{~s}^{-1}\right)\end{array}$ & $\begin{array}{l}\text { Observation } \\
\text { Date }\end{array}$ & Reference \\
\hline $476 \pm 24$ & $1.8 \pm 0.5$ & 1971 Nov 20 & Johnson and Haymes 1973 \\
\hline $530 \pm 11$ & $0.80 \pm 0.23$ & 1974 Apr 2 & Haymes et al. 1975 \\
\hline $511 \ldots \ldots$ & $4.18 \pm 1.56$ & 1977 Feb 14, 17 & Albernhe et al. 1981 \\
\hline $510.7 \pm 0.5$ & $1.22 \pm 0.22$ & 1977 Nov 11 & $\begin{array}{l}\text { Leventhal, MacCallum, and Stang } \\
1978\end{array}$ \\
\hline $511 \ldots \ldots \ldots \ldots$ & $\begin{array}{c}2.35 \pm 0.71 \\
\text { or } \\
1.24 \pm 0.43\end{array}$ & 1979 Apr 15 & Leventhal et al. 1980 \\
\hline $510.90 \pm 0.25$ & $1.85 \pm 0.21$ & 1979 Oct ) & \\
\hline $510.1^{\mathrm{a}} \ldots \ldots \ldots$ & $0.65 \pm 0.27$ & $1980 \mathrm{Mar}\}$ & this Letter \\
\hline
\end{tabular}

\footnotetext{
${ }^{a}$ Because of the low statistical significance of this observation, the line center energy for the net spectrum was assumed to be equal to the line center energy for the source-plus-background spectrum.
}

fall data. By 1980 spring, radiation damage due to the cumulative effect of charged particle bombardment had broadened the resolution to $5.7 \mathrm{keV}$ (Mahoney, Ling, and Jacobson 1981). Except for line broadening, radiation damage does not affect the results presented below. For example, the observed intensity of an on-board $\mathrm{K}^{40}$ calibration line at $1460 \mathrm{keV}$ remained constant to within $3.2 \pm 3.2 \%$ between 1979 fall and 1980 spring. The intensity of continuum emission between $70 \mathrm{keV}$ and $180 \mathrm{keV}$ from the Crab Nebula was observed to remain constant to within $2.1 \pm 2.3 \%$.

Collimation was provided by cylindrical holes in the shield piece directly in front of the germanium crystals. The average effective field of view for all four detectors at $511 \mathrm{keV}$ was $35^{\circ} \mathrm{FWHM}$, and the systematic alignment uncertainty was 0.5 . Sidelobes due to the shield collimation holes gave the collimator response curve a distinctly nontriangular shape. A detailed description of the HEAO 3 Gamma-Ray Spectrometer is given by Mahoney et al. (1980).

\section{OBSERVATIONS AND DATA ANALYSIS}

$H E A O 3$ was launched into a near-circular orbit of $500 \mathrm{~km}$ altitude and $43^{\circ} .6$ inclination on 1979 September 20. During the "nominal" scans, which will be referred to later, the viewing direction scanned a great circle which intercepted the galactic plane within approximately $10^{\circ}$ of the galactic center at an inclination of $30^{\circ}$. The Gamma-Ray Spectrometer also scanned precisely along the galactic plane for extended periods in 1979 fall and 1980 spring. Starting on 1979 September 27,14 consecutive days were spent in the "galactic plane scan" mode. Between 1980 March 4 and April 4, a total of 15 days were spent in the galactic plane scan mode, interwoven on a weekly cycle with periods of nominal scans.

Because of the large in-orbit variability of the 511 $\mathrm{keV}$ background line and the importance of the mea- surement of an astronomical $511 \mathrm{keV}$ emission feature, three different data analysis methods and computer program packages were used. The results of the three methods were found to be mutually consistent. Two of the methods emphasized treatments of the variable background rate. The first approach corrected for the magnetic latitude effect by an empirical fit to the 511 $\mathrm{keV}$ background rate as a function of the McIlwain $L$-parameter (all data presented here were accumulated at $L \leq 1.6)$. The second approach subtracted the background on a scan-by-scan basis; a weighted average over many scans then produced the final result.

The present results are based on the third analysis approach, in which 8192 channel gain-normalized pulseheight spectra were accumulated separately for each scan angle bin for each of the four detectors as well as a four-detector sum. A net $511 \mathrm{keV}$ signal was obtained by computing the difference between data in a group of pulse-height channels which included the $511 \mathrm{keV}$ line and the average of data from nearby pulse-height channel intervals above and below the line. The resulting one-dimensional array contained the net instrumental plus astronomical $511 \mathrm{keV}$ line emission as a function of galactic longitude or scan angle and was then analyzed with a fitting program which searches for features similar to the instrument's one-dimensional collimator response pattern. Figure $1 a$ shows the best fit to the 1979 fall galactic-longitude distribution of the instrumental and astronomical $511 \mathrm{keV}$ line flux. The best-fitting curve, with $\chi^{2} /$ d.o.f. $=1.36$, corresponds to a flux level of $(1.85 \pm 0.21) \times 10^{-3}$ photons $\mathrm{cm}^{-2} \mathrm{~s}^{-1}$ (all errors quoted are derived from counting statistics only at $68 \%$ confidence). The signal, $37 \%$ above the average background level, is centered at $l^{\mathrm{II}}=3: 9 \pm 4: 0$, if a point source in the galactic plane is assumed. If an extended source with constant source density in longitude in the galactic plane is assumed, then the best-fit response yields a source center at $l^{\mathrm{II}}=3.5 \pm 4.0$ and a source 


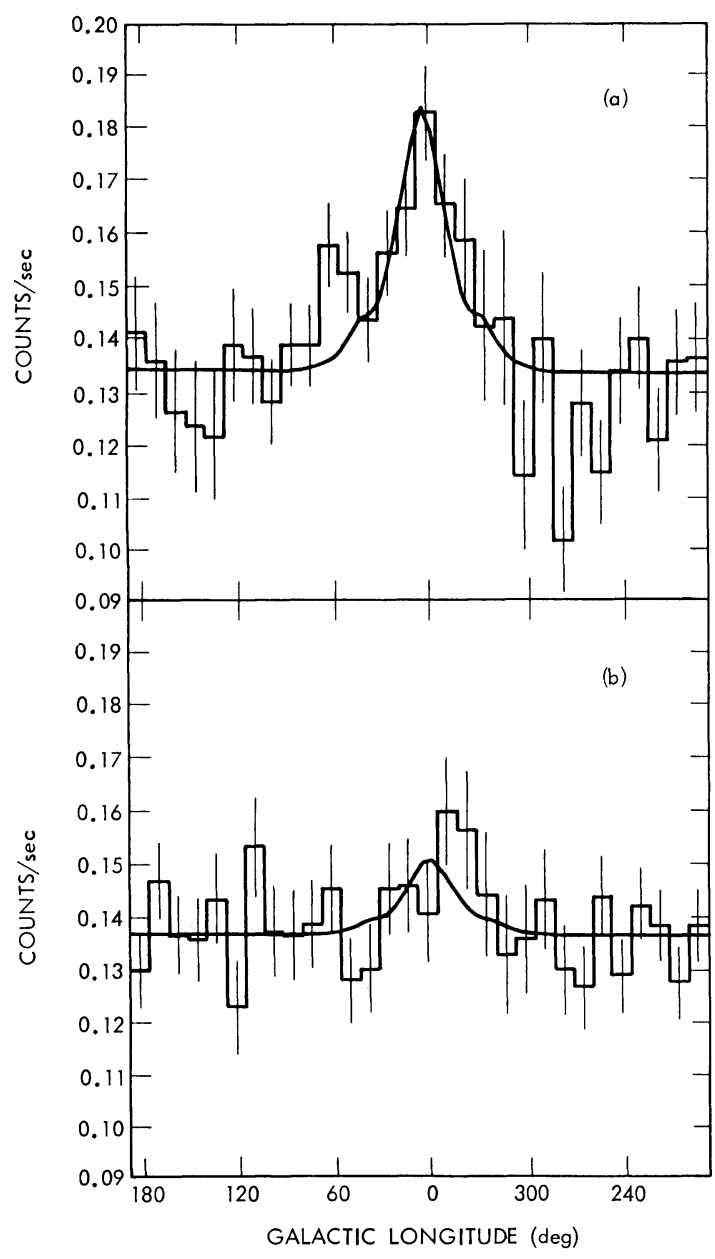

Fig. 1.- Net instrumental and astronomical $511 \mathrm{keV}$ line flux as a function of galactic longitude for the $(a) 1979$ fall galactic plane scan and $(b)$ all 1980 spring scans. An on-line bandpass of $8.54 \mathrm{keV}$ was used for the 1979 fall data, and $13.42 \mathrm{keV}$ for the 1980 spring data. The solid lines show the best-fitting background level, source intensity, and source galactic longitude for a point source.

extent of $19^{\circ} \pm 8^{\circ}$ with $\chi^{2} /$ d.o.f. $=1.34$. (At the $90 \%$ confidence level, all source extents from $0^{\circ}$ through $45^{\circ}$ are consistent with the data.) Since the addition of a variable intrinsic source width did not produce any statistically significant improvement of the fit, a point source in the galactic plane was assumed for all other fits.

Figure $1 b$ shows the equivalent data for the 1980 spring galactic plane and nominal scans for the net 511 $\mathrm{keV}$ flux together with the best fit to a point source at the galactic center. The curve shown corresponds to a flux of $(0.65 \pm 0.27) \times 10^{-3}$ photons $\mathrm{cm}^{-2} \mathrm{~s}^{-1}$. Between the 1979 fall and 1980 spring observations, the $511 \mathrm{keV}$ flux decreased by $(1.20 \pm 0.35) \times 10^{-3}$ photons $\mathrm{cm}^{-2} \mathrm{~s}^{-1}$. The statistical likelihood of an upward or downward change in flux level by $3.5 \sigma$ is $5.0 \times 10^{-4}$ for a normal distribution.

In order to derive a net spectrum of the galactic center, data collected while viewing regions away from the galactic center or known prominent gamma-ray emitters $(97<l<139$ and $217<l<265)$ were subtracted via linear interpolation from data collected while the galactic center was within the field of view $(331<l$ $<19$ ). A fit to the net galactic center spectrum from the 1979 fall galactic plane scan is shown in Figure 2. Because of resolution broadening and the lower intensity, spectral parameters for the 1980 spring data have larger uncertainties. The line in the 1979 fall spectrum is centered at $510.90 \pm 0.25 \mathrm{keV}$ and has an observed width of $3.13 \pm 0.57 \mathrm{keV}$ FWHM. With an instrumental resolution of $2.72 \mathrm{keV}$ FWHM, this corresponds to an intrinsic line width of $1.6(+0.9,-1.6) \mathrm{keV}$ FWHM.

Positronium decay will produce a photon continuum from the triplet state in addition to the $511 \mathrm{keV}$ line from the singlet state (Leventhal 1973; Leventhal, MacCallum, and Stang 1978). Using the predicted lineto-continuum flux ratio and the observed $511 \mathrm{keV}$ line flux, a continuum intensity of $I_{c} \sim 0.9 \times 10^{-3}$ counts $\mathrm{s}^{-1}$ channel $^{-1}$ would be expected for the case of positronium decay. This expected $I_{c}$ value is in fact an upper limit, since direct positron annihilation increases the line-to-continuum flux ratio depending on the density, temperature, and state of ionization of the annihilation region (Bussard, Ramaty, and Drachman 1979). The apparent continuum flux of $\approx 1.5 \times 10^{-3}$ counts $\mathrm{s}^{-1}$ channel $^{-1}$, which appears in Figure 2 below and above the $511 \mathrm{keV}$ line, is an artifact of the simple linear background interpolation technique which was used

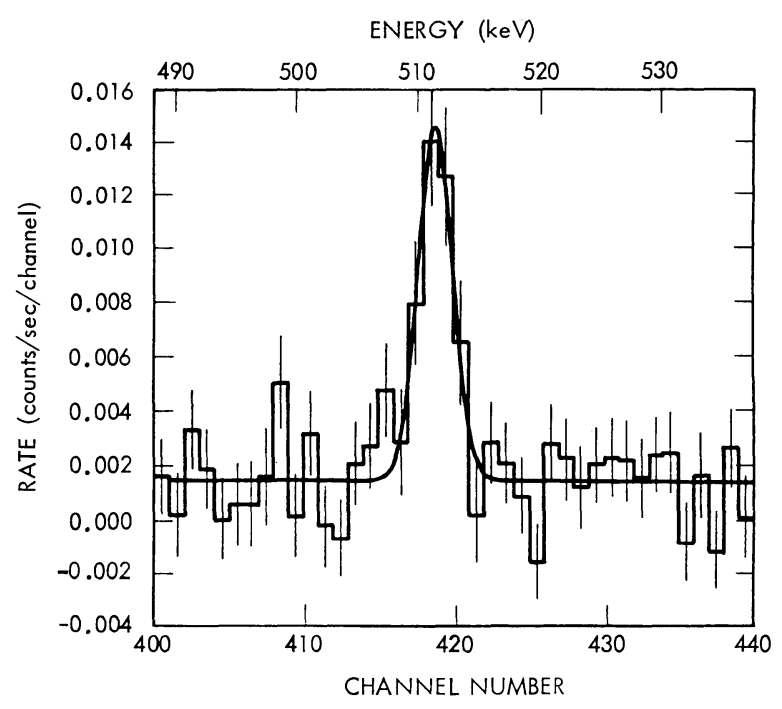

Fig. 2.- Net galactic center spectrum from the 1979 fall galactic plane scan. The line emission signal is centered at 510.90 $\pm 0.25 \mathrm{keV}$. 
'here; it prevents conclusions being drawn about the 1olexistence of the positronium decay spectrum. The results -of more sophisticated spectrum analysis near $511 \mathrm{keV}$ and at other energies will be presented elsewhere.

\section{CONCLUSIONS}

We have presented evidence for the existence of time varying, unshifted, narrow $511 \mathrm{keV}$ line emission from the vicinity of the galactic center. Uncertainties exist regarding the spatial extent of the feature as well as its centroid. Nevertheless, all data are also consistent with emission from a single point source located at the galactic center. This interpretation would require a source luminosity at $511 \mathrm{keV}$ of $L=2 \times 10^{37} \mathrm{ergs} \mathrm{s}^{-1}$ and a positron annihilation rate of $\approx 10^{43} \mathrm{~s}^{-1}$.

The range of $511 \mathrm{keV}$ line flux levels reported from earlier observations (Table 1) is consistent with the 1979 fall observations. In the light of the flux variation reported here, the earlier observations may also be viewed as suggestive of flux variability. The narrow spatial extent is inconsistent with the cosmic-ray origin of positrons, as proposed by Ramaty, Stecker, and Misra (1970). Other difficulties with the cosmic-ray hypothesis have been described by Ramaty, Kozlovsky, and Lingenfelter (1979) and by Ramaty and Lingenfelter (1981). The narrow spatial extent or point source nature of the observed emission is also inconsistent with distributions of supernovae of types I or II, as well as of pulsars and of novae. If it is a result of a single point source, then the observed variability suggests a size of the order of $10^{18} \mathrm{~cm}$ and requires positron annihilation in a medium of density $10^{4} \mathrm{~cm}^{-3}$ to $10^{6} \mathrm{~cm}^{-3}$, depending on the initial positron energy and ambient temperature (Ramaty and Lingenfelter 1981). Regardless of how the positrons have been generated, the narrow observed $511 \mathrm{keV}$ line width implies that the annihilation region is partially ionized and has a temperature $<10^{5} \mathrm{~K}$ (Bussard, Ramaty, and Drachman 1979). This annihilation region may be found in the clouds of ionized gas within Sgr A West. A variable source of positrons which could generate the $\sim 10^{43}$ positron-electron pairs $\mathrm{s}^{-1}$ required by the observations (Ramaty and Lingenfelter 1981) might be a massive black hole at the galactic center which had been suggested by infrared observations (Lacy et al. 1980).

We wish to thank J. Bradley, J. Brown, A. Dunklee, B. Gokhman, and R. Radocinsky for the development of the processing and analysis software; B. Teegarden, R. Ramaty, and R. Lingenfelter for suggestions on the data handling and interpretation; and M. Neugebauer and A. Metzger for valuable comments on this Letter. We also express our appreciation to the many persons who have worked with great dedication during the long development of this successful experiment, notably $\mathbf{R}$. Jorgensen at the Jet Propulsion Laboratory, and J. Lindner and his colleagues at TRW Systems. This work was carried out under NASA contract NAS7-100 at the Jet Propulsion Laboratory, California Institute of Technology, and under NASA grant NGR 05-002-160 at the California Institute of Technology. W. A. W. is a NAS/National Research Council Resident Research Associate.

\section{REFERENCES}

Albernhe, F., Leborgne, J. F., Vedrenne, G., Boclet, D., Durouchoux, P., and da Costa, J. M. 1981, Astr. Ap., 94, 214.

Bussard, R. W., Ramaty, R., and Drachman, R. J. 1979, Ap. J. 228, 928 .

Haymes, R. C., Walraven, G. D., Meegan, C. A., Hall, R. D., Djuth, F. T., and Shelton, D. M. 1975, Ap. J., 201, 593.

Jacobson, A. S., Ling, J. C., Mahoney, W. A., and Willett, J. B. 1978, Gamma-Ray Spectroscopy in Astrophysics, ed. T. L. Cline and R. Ramaty (NASA TM-79619), p. 228.

Johnson, W. N., and Haymes, R. C. 1973, Ap. J., 184, 103.

Lacy, J. H., Townes, C. H., Geballe, T. R., and Hollenbach, D. J. 1980, Ap. J., 241, 132.

Leventhal, M. 1973, Ap. J. (Letters), 183, L147.

Leventhal, M., MacCallum, C. J., Huters, A. F., and Stang, P. D. 1980, Ap. J., 240, 338.

Leventhal, M., MacCallum, C. J., and Stang, P. D. 1978, Ap. J. (Letters), 225, L11.

Leventhal, M., MacCallum, C. J., and Watts, A. C. 1977, Nature, 225, 696 .

Allan S. Jacobson, James C. Ling, William A. Mahoney, Guenter R. Riegler, William A. Wheaton, and James B. Willett: M. S. 169-327, Jet Propulsion Laboratory, California Institute of Technology, Pasadena, CA 91109

Thomas A. Prince: California Institute of Technology, 220-47, Pasadena, CA 91125
Lingenfelter, R. E., Higdon, J. C., and Ramaty, R. 1978, GammaRay Spectroscopy in Astrophysics, ed. T. L. Cline and R. Ramaty (NASA TM-79619), p. 252

Mahoney, W. A., Ling, J. C., and Jacobson, A. S. 1981, Nucl. Instr. Methods, in press.

Mahoney, W. A., Ling, J. C., Jacobson, A. S., and Tapphorn, R. 1980, Nucl. Instr. Methods, 178, 363

Mazets, E. P., Golenetskii, S. V., Aptekar, R. L., Guryan, Yu. A., and Il'inskii, V. N. 1980, Academy of Science of the USSR preprint, Leningrad (Loffe Physico-Tech. Inst. preprint 687)

Ramaty, R., and Lingenfelter, R. E. 1981, Phil. Trans. Roy. Soc. London, in press.

Ramaty, R., Kozlovsky, B., and Lingenfelter, R. E. 1979, Ap. J. Suppl., 40, 487.

Ramaty, R., Stecker, F. W., and Misra, D. 1970, J. Geophys. Res., 75, 1141 .

Sturrock, P. A. 1971, Ap. J., 164, 529.

Teegarden, B. J., and Cline T. L. 1980, Ap. J. (Letters), 226, L67. 